\title{
Global research hotspots and research trends on idiopathic pulmonary fibrosis: a bibliometric and visualization analysis
}

\author{
Yue $\mathrm{Ma}^{1}$, Renyi Zhou ${ }^{2}$, Qiong $\mathrm{Wu}^{3}$ \\ ${ }^{1}$ Department of Pulmonary and Critical Care Medicine, Shengjing Hospital of China Medical University, Shenyang, China; ${ }^{2}$ Department of \\ Orthopaedics, The First Hospital of China Medical University, Shenyang, China; ${ }^{3}$ Department of Pediatrics, Shengjing Hospital of China Medical \\ University, Shenyang, China \\ Contributions: (I) Conception and design: All authors; (II) Administrative support: Q Wu; (III) Provision of study materials or patients: Y Ma, Q Wu; \\ (IV) Collection and assembly of data: Y Ma, R Zhou; (V) Data analysis and interpretation: All authors; (VI) Manuscript writing: All authors; (VII) \\ Final approval of manuscript: All authors. \\ Correspondence to: Qiong Wu. Department of Pediatrics, Shengjing Hospital of China Medical University, No. 36 Sanhao Street, Heping district, \\ Shenyang 110004, China. Email: wuqiong@cmu.edu.cn.
}

Background: Idiopathic pulmonary fibrosis (IPF) is a life-threatening disease, the progression of which
current drug therapy cannot reverse. This study analyzed current research hotspots and future research
trends in IPF through bibliometric methods, with the aim of providing a reference for new therapeutic
strategies.
Methods! Publications on IPF obtained from the Web of Science Core Collection database, The Literature
Metrology Online Analysis Platform, and CiteSpace were used to analyze publication characteristics.
VOSviewer was used to conduct keywords co-occurrence analysis and analyze research hotspots.
Results: A total of 7,016 publications related to IPF were identified from 2011 to 2020. The most
contributions were from the USA and the five research institutions with the largest number of publications
were all from that country. The American fournal of Respiratory and Critical Care Medicine was the most
cited journal and had an incontrovertible academic impact with five of the top 10 high-cited references
published in this journal. G Raghu was the academic authority in this domain in terms of both the number of
publications and the most citations. By analyzing keywords, we identified three IPF research hotspot clusters,
which are "clinical research", "pathogenesis research" and "diagnosis research" respectively. Conclusions: We evaluated all publications concerning IPF research in the past decade through bibliometric analysis. The current research hotspot in this field is drug therapy for the condition using nintedanib and pirfenidone. Future research will focus on conducting multi-center randomized controlled trials to explore and evaluate new therapeutic drugs for IPF. It is hoped that this study can provide information and data support for further research and the development of new therapeutic drugs.

Keywords: Bibliometric analysis; hotspots; idiopathic pulmonary fibrosis (IPF); nintedanib; pirfenidone

Submitted Jul 06, 2021. Accepted for publication Jul 28, 2021.

doi: 10.21037/apm-21-1836

View this article at: https://dx.doi.org/10.21037/apm-21-1836

\section{Introduction}

Idiopathic pulmonary fibrosis (IPF) is a chronic, progressive, fibrotic interstitial pneumonia of unknown etiology (1), and is one of the most common idiopathic interstitial pneumonia seen clinically. Although previously considered rare, its morbidity has shown an upward trend in recent years (2). The main manifestations are dry cough, and progressive and aggravated dyspnea, accompanied by restricted ventilation dysfunction and gas exchange dysfunction (3), which lead to hypoxia and even respiratory failure. Its chest high-resolution 


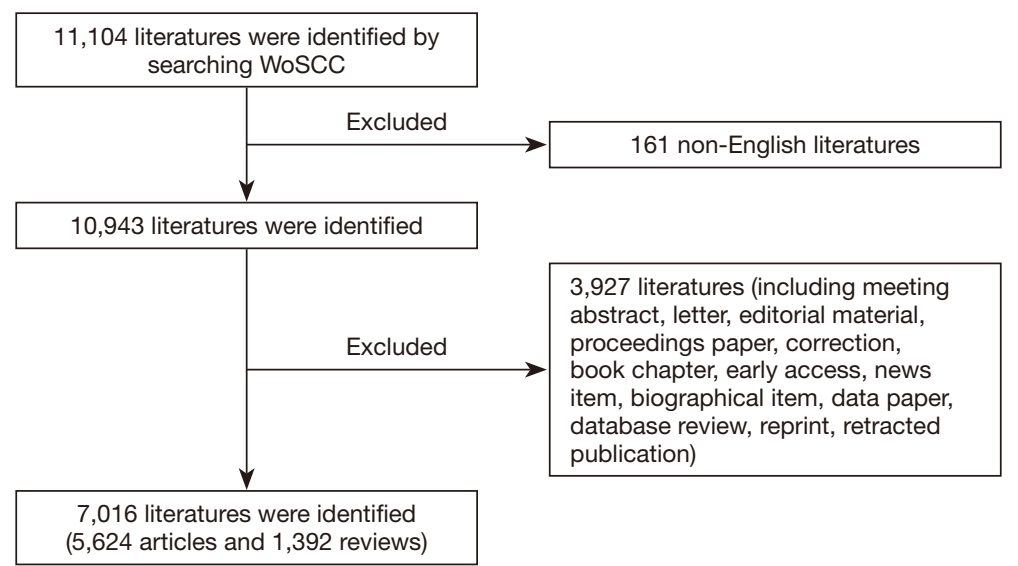

Figure 1 Flow chart of literature screening.

CT (HRCT) is characterized by subpleural and basal predominant honeycombing, with or without peripheral traction bronchiectasis (1). IPF has a poor prognosis, with a median survival time of only 3-5 years after diagnosis (4), although some patients may experience acute deterioration of respiratory function in a short period of time leading to death.

As the pathogenesis of IPF is unclear, and drug therapy cannot reverse the progression of the disease, most patients continue to deteriorate, and their quality of life is seriously reduced, causing a serious economic burden to patients, their families and communities. For these reasons, determining the pathogenesis of IPF and mechanisms by which the disease can be treated is crucial.

Bibliometrics is a discipline that uses mathematical and statistical methods to quantitatively analyze information (5). It can be used to assess the contributions of countries, institutions, journals, and authors to a particular research topic, and can determine research hotspots and forecast research trends in a certain field. While information gleaned from bibliometrics can also provide the basis for the formulation of clinical guidelines $(6,7)$, there is currently no bibliometric study on IPF. The aim of this study was to analyze literature about IPF through information visualization software, and intuitively showed the importance of countries, institutions, authors and keywords in the network visualization map, thus summarize the current academic authority and achievements, identify hotspots and future directions, and provide a reference for future investigations.

\section{Methods}

\section{Data collection}

We performed an online search of the Web of Science Core Collection database on March 1, 2021. The search formula was set to TS = (idiopathic pulmonary fibrosis), time span $=(2011-2020)$, and language $=$ English. Only articles and reviews were selected (Figure 1) and the title, abstract, keywords, "country", "institution”, "authors”, "journal", and "references" were collected.

\section{Data analysis}

The Literature Metrology Online Analysis Platform (https://bibliometric.com/), CiteSpace v.5.7.R5(64-bit)W, and VOSviewer were used to perform bibliometric analysis, and the annual publication numbers were determined through the Literature Metrology Online Analysis Platform. CiteSpace is an optimal method for collaborative network analysis of productive countries, institutions, and authors, and can also be used for cited journals, cited references, and cited authors analysis. CiteSpace was used for keywords burst detection to trace research hotspots and research trends, and to perform "time slicing" function, by setting the "years per slice" to " 1 ", and the "top N" to " 50 ", to obtain the top 50 publications in a year in a single file. In this study, the node size represents the number of publications or the frequency of being cited. Centrality is an index to measure the importance of nodes in a network. A higher centrality indicates that a node is more important 
in the network, and the purple rings outside the circle refer to high centrality. In CiteSpace, the timeline is displayed above the network visualization map, and the different link colors represent the different times. VOSviewer can classify keywords into different clusters according to cooccurrence analysis, and the different colors represent different clusters.

\section{Results}

In total, 7,016 publications (including 5,624 articles and 1,392 reviews) met the inclusion criteria from 2011 to 2020 (Figure 1). Figure 2 shows the total number of publications annually, where the number of papers in IPF research maintained a gradual increasing trend from 2011 to 2020.

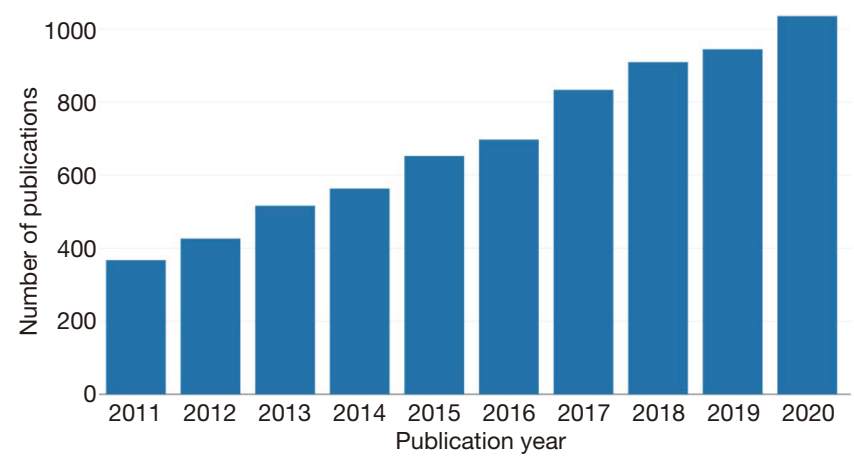

Figure 2 Number of annual publications.

\section{Analysis of leading countries and institutions}

The country collaboration network of IPF research is shown in Figure 3, and the top 10 countries contributing to it are shown in Table 1. The USA contributed the most publications [2,126], followed by Japan [898], China [833], England [539], and Germany [484]. Centrality is an index to measure the importance of nodes in a network, and it was apparent that the USA (centrality $=0.2$ ) was at the heart of the network, followed by Germany (centrality $=0.18$ ), and Japan (centrality $=0.12$ ). A higher centrality in a collaboration network means more frequent cooperation.

The institution cooperation network is visualized in Figure 4, which shows the top 10 institutions as including the University of Michigan [193], National Jewish Health [181], University of California, San Francisco [175], Mayo clinic [163], and University of Pittsburgh [160] (Table 1), which are all located in the USA. Among them, the highest ranking of centrality were the University of California, San Francisco (centrality $=0.15$ ) and National Jewish Health (centrality $=0.12$ ), respectively.

\section{Analysis of cited journals and cited references}

Table 2 lists the top 10 cited journals. The highest cited journal was the American Fournal of Respiratory and Critical Care Medicine, which was cited 4,787 times, followed by the European Respiratory Fournal [3,415] and Chest [3,086]. According to the 2019 standard of the JCR, seven journals

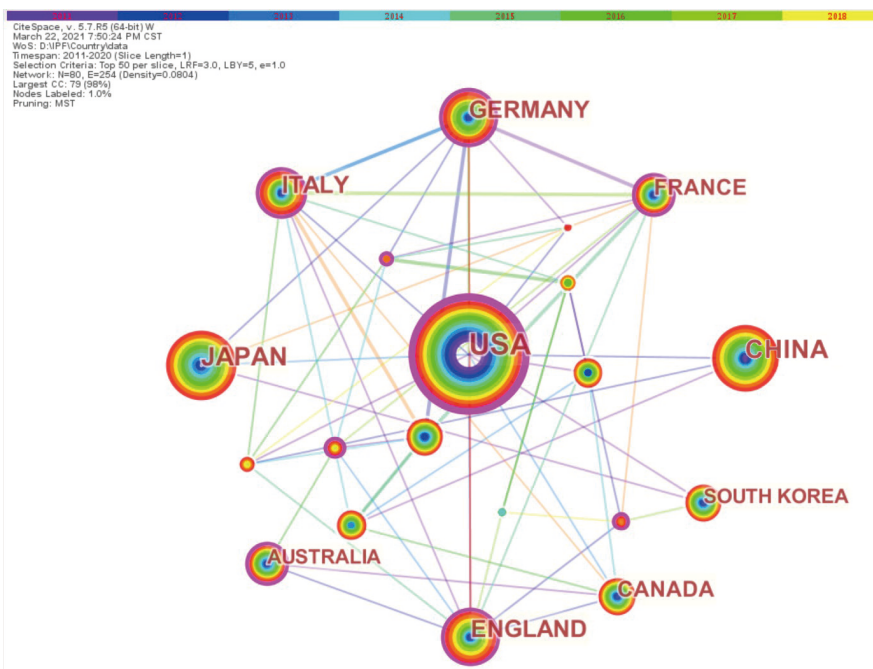

Figure 3 Cooperation network of the productive countries. The node size represents the number of publications of the country. The purple rings outside the circle refer to high centrality. 
Table 1 Top 10 countries and institutions contributing to publications

\begin{tabular}{|c|c|c|c|c|c|c|}
\hline Rank & \multicolumn{3}{|c|}{ Countries } & \multicolumn{3}{|c|}{ Institutions } \\
\hline 1 & USA & 2,126 & 0.2 & Univ Michigan & 193 & 0.05 \\
\hline 2 & Japan & 898 & 0.12 & Natl Jewish Hlth & 181 & 0.12 \\
\hline 3 & China & 833 & 0.06 & Univ Calif San Francisco & 175 & 0.15 \\
\hline 5 & Germany & 484 & 0.18 & Univ Pittsburgh & 160 & 0.09 \\
\hline 6 & Italy & 434 & 0.12 & Royal Brompton Hosp & 142 & 0.11 \\
\hline 7 & France & 302 & 0.11 & Univ Washington & 129 & 0.08 \\
\hline 8 & Canada & 292 & 0.12 & Univ Colorado & 112 & 0.05 \\
\hline
\end{tabular}

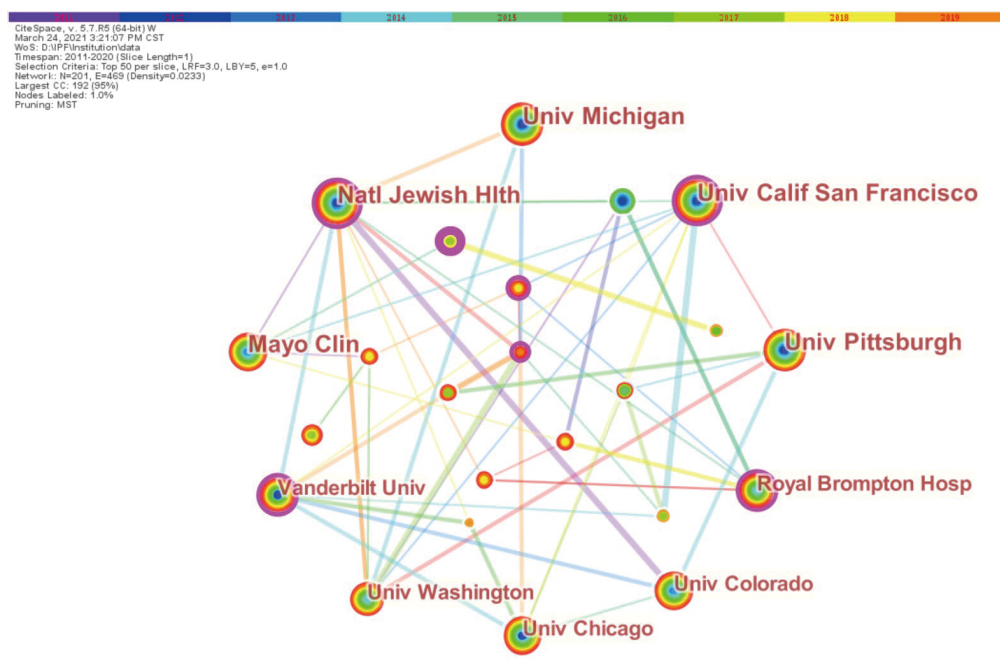

Figure 4 Cooperation network of the productive institutions. The node size represents the number of publications of the institution.

were sorted to Q1, and three were sorted to Q2. The New England fournal of Medicine had the highest impact factor (74.699), followed by the American Fournal of Respiratory and Critical Care Medicine (17.452), and the European Respiratory Fournal (12.339).

Details of the top 10 cited references are provided in Table 3. The publication titled "An official ATS/ERS/FRS/ ALAT statement: idiopathic pulmonary fibrosis: evidence-based guidelines for diagnosis and management" was the most cited, being cited 913 times. The second most cited was "Efficacy and safety of nintedanib in idiopatbic pulmonary fibrosis", being cited 620 times, and "A phase 3 trial of pirfenidone in patients with idiopathic pulmonary fibrosis" was the third (604 times). In the top 10 cited references, five publications were from the American Fournal of Respiratory and Critical Care Medicine, three were from The New England Fournal of Medicine, and two were from the Lancet.

\section{Analysis of authors and co-cited authors}

The author collaboration network of IPF is shown in Figure 5. The top 10 authors with the most publications include Wells AU [115], Maher TM [96], Raghu G [93], Collard HR [86], and Brown KK [84] (Table 4). 
Table 2 Top 10 cited journals

\begin{tabular}{|c|c|c|c|c|}
\hline Rank & Cited journals & Cited counts & JCR [2019] & IF [2019] \\
\hline 2 & European Respiratory Journal & 3,415 & Q1 & 12.339 \\
\hline 3 & Chest & 3,086 & Q1 & 8.308 \\
\hline 4 & The New England Journal of Medicine & 2,595 & Q1 & 74.699 \\
\hline 6 & PLoS One & 2,229 & Q2 & 2.740 \\
\hline 7 & American Journal of Respiratory Cell and Molecular Biology & 2,040 & Q1 & 5.373 \\
\hline 8 & American Journal of Physiology-Lung Cellular and Molecular Physiology & 1,872 & Q2 & 4.418 \\
\hline 9 & Journal of Clinical Investigation & 1,764 & Q1 & 11.864 \\
\hline
\end{tabular}

Table 3 Top 10 cited references

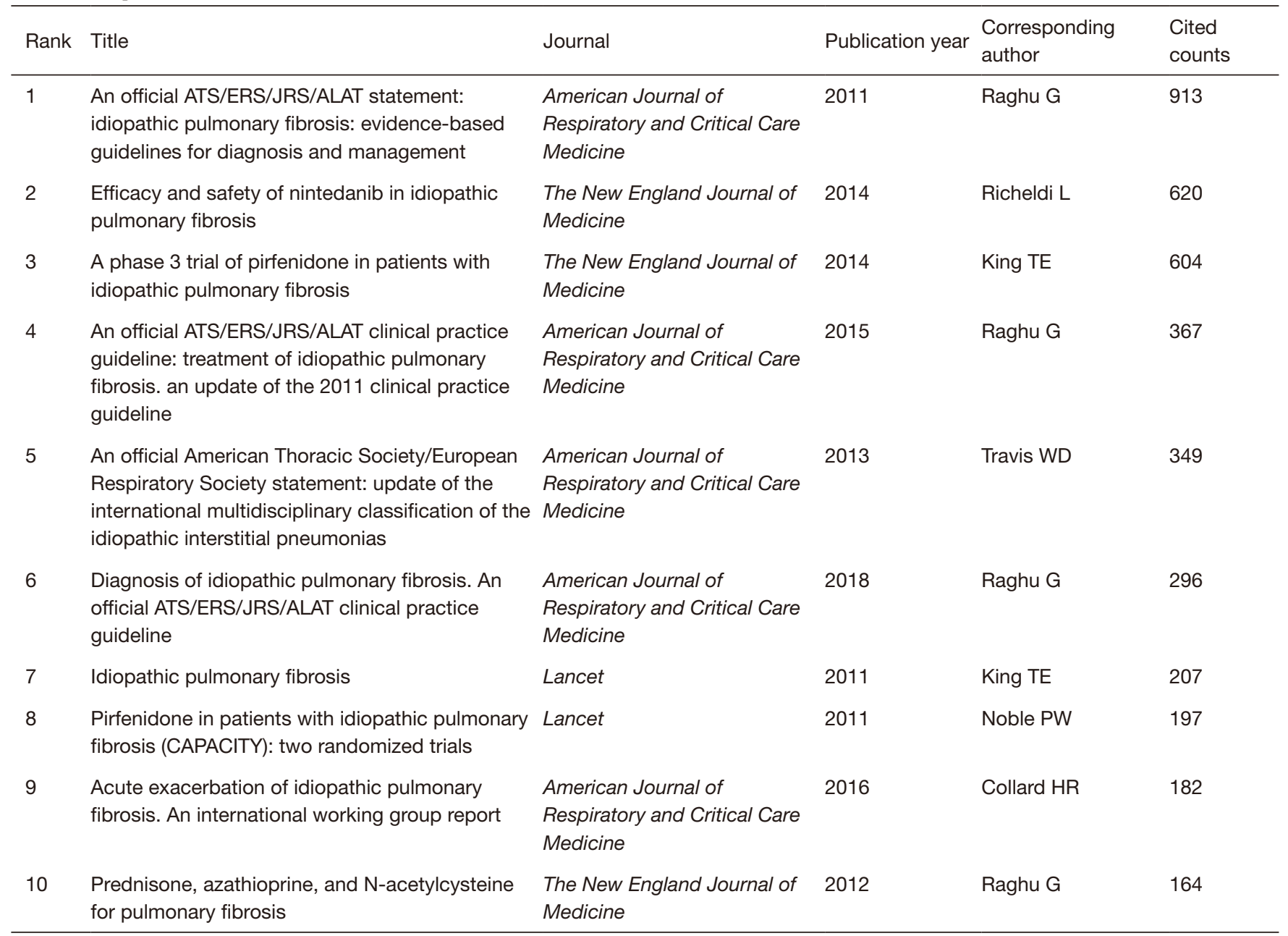




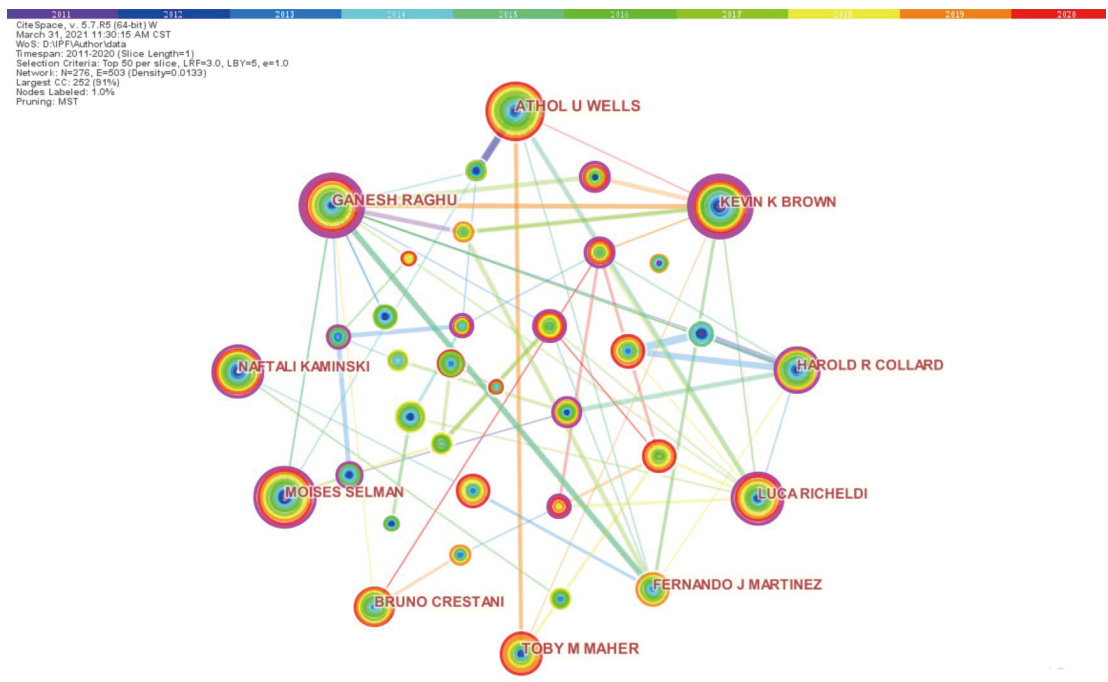

Figure 5 Cooperation network of the productive authors. The node size represents the number of papers published by the author. The link color represents the earliest date of their collaboration.

Table 4 Top 10 productive authors and co-cited authors contributing to publications

\begin{tabular}{|c|c|c|c|c|c|}
\hline Rank & Authors & Article counts & Centrality & Co-cited authors & Cited counts \\
\hline 2 & Maher TM & 96 & 0.08 & King TE & 1,528 \\
\hline 3 & Raghu G & 93 & 0.21 & Richeldi L & 1,024 \\
\hline 4 & Collard HR & 86 & 0.14 & Travis WD & 820 \\
\hline 6 & Selman M & 81 & 0.11 & Collard HR & 750 \\
\hline 7 & Richeldi L & 74 & 0.16 & Ley B & 710 \\
\hline 8 & Kaminski N & 73 & 0.13 & Noble PW & 589 \\
\hline 9 & Martinez FJ & 67 & 0.06 & Flaherty KR & 585 \\
\hline
\end{tabular}

Among them, Raghu and Brown had high centrality and collaborated extensively with other authors, and although Wells and Maher published more papers, they had low centrality and less collaboration with other authors. Figure 5 shows the earliest collaborations were in 2015 (the link is shown as light green).

Co-cited authors networks are visualized in Figure 6. Of the 10 most cited authors, Raghu ranks first, with 2,853 citations, followed by King [1,528], and Richeldi [1,024] (Table 4). The earliest link of Raghu is shown in purple (in 2011), while that of Richeldi is green (in 2016), implying he is more recent author.

\section{Analysis of research hotspots and research trends}

Keywords were identified in 7,016 documents and analysed by VOSviewer, and the network visualization map shows their frequency of occurrence (Figure 7). The analysis identified 217 keywords that appeared more than 218 times and divided them into three clusters: cluster 1 (clinical research, red); cluster 2 (pathogenesis research, green); cluster 3 (diagnosis research, blue). In cluster 1, the highfrequency keywords were "patient" [15,676], "IPF" [10,737], "idiopathic pulmonary fibrosis" [6,718], "study" [5,961], and "treatment" $[4,142]$. In cluster 2, the keywords that 


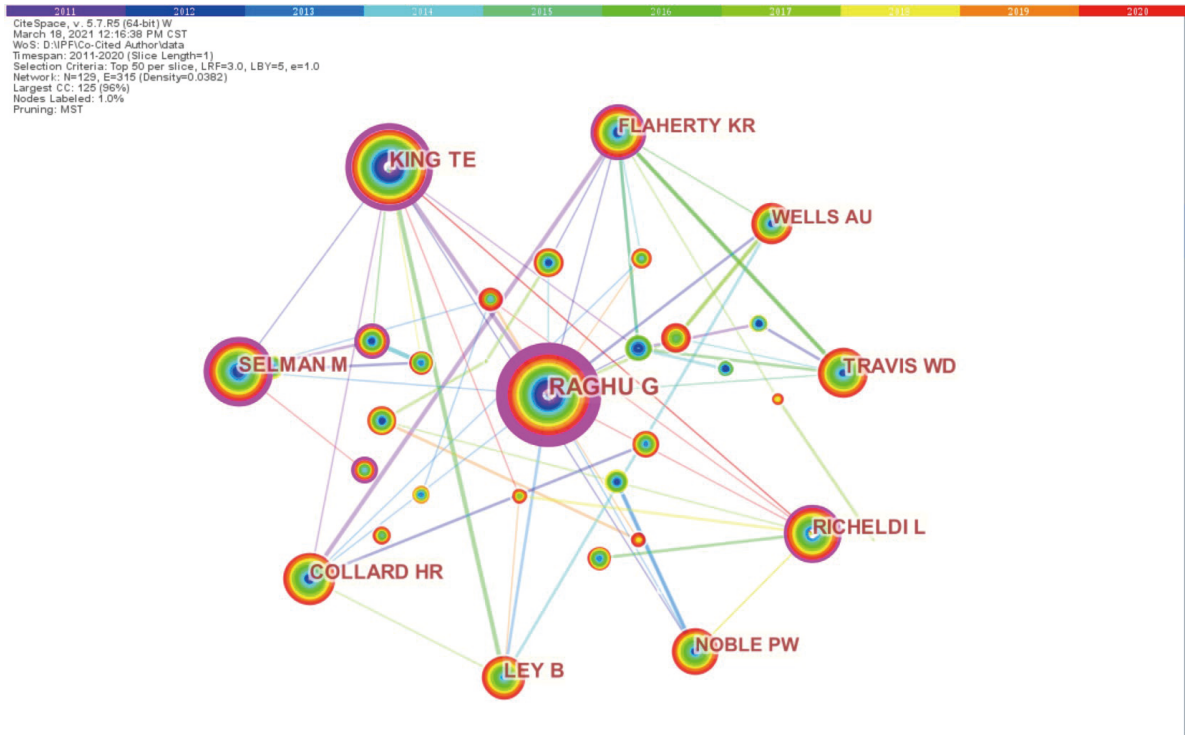

Figure 6 Cooperation network of the co-cited authors. The size of the node represents the frequency the author was cited. The link color represents the earliest time the author was co-cited.

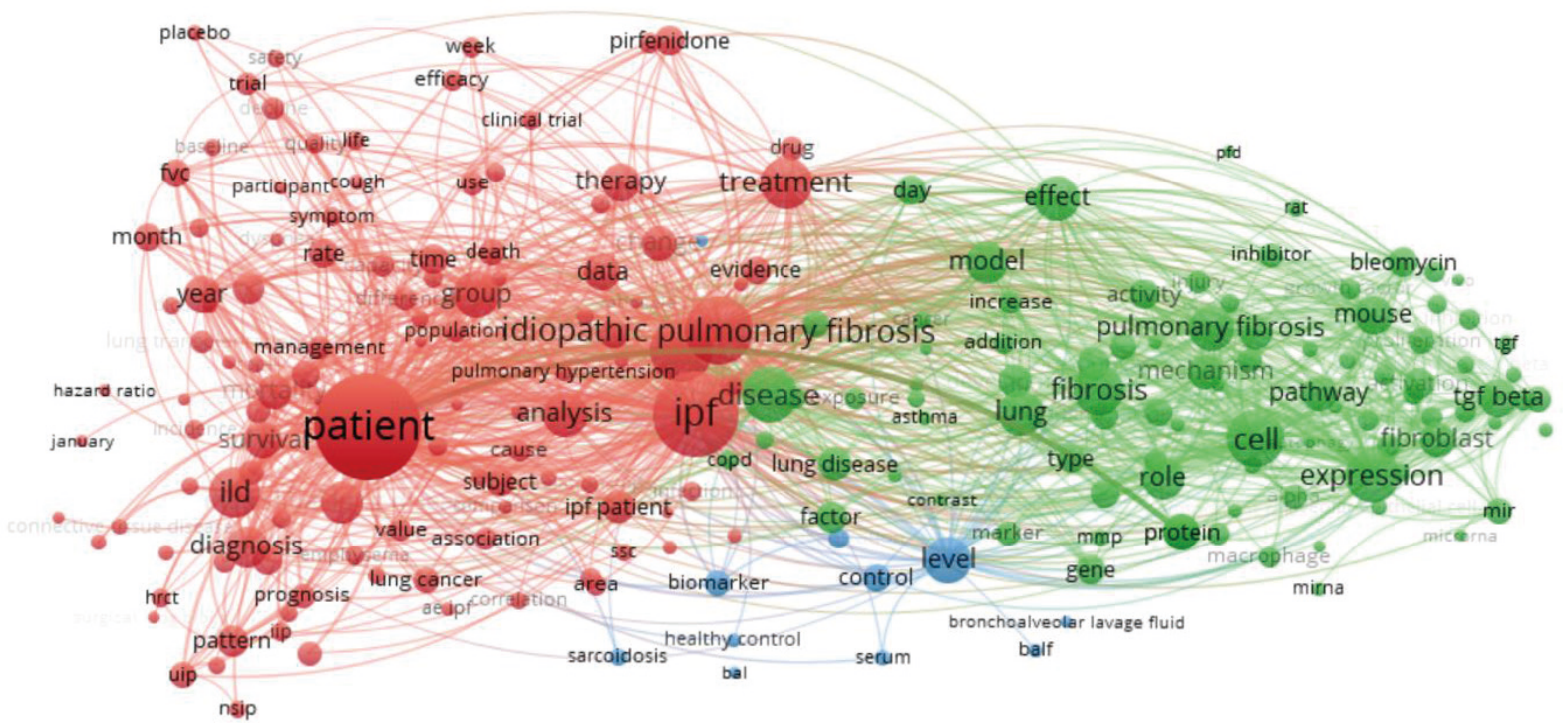

Figure 7 Network visualization map of keywords. The different colors represent the different clusters. Large circles indicate the keywords of high frequency.

appeared frequently were "cell" [4,908], "disease" [4,539], "expression" [4,204], "fibrosis" [3,775], and "pulmonary fibrosis" [3,136], and in cluster 3, the primary keywords were "level" [3,224], "control" [1,155], "biomarker" [998], "concentration" [707], and "sarcoidosis" [445]. Table S1 shows details of the keywords.

Figure 8 shows the top 25 keywords with the strongest citation bursts from 2011 to 2020 by CiteSpace, which was used to analyze the temporal trend of research hotspots shift.

\section{Discussion}

The incidence of IPF seems to be increasing in recent years. In this study, we applied information visualization 
Top 25 Keywords with the Strongest Citation Bursts

\begin{tabular}{lccll}
\multicolumn{1}{c}{ Keywords } & Strength & Begin & End & 2011 - 2020 \\
Growth factor beta & 22.41 & $\mathbf{2 0 1 1}$ & 2016 & \\
Alveoliti & 16.67 & $\mathbf{2 0 1 1}$ & 2013 & \\
Feature & 15.50 & $\mathbf{2 0 1 1}$ & 2013 & \\
Sarcoidosis & 13.13 & $\mathbf{2 0 1 1}$ & 2013 & \\
Endoplasmic reticulum stress & 11.61 & $\mathbf{2 0 1 1}$ & 2012 & \\
Receptor & 11.16 & $\mathbf{2 0 1 1}$ & 2014 & \\
Myofibroblas & 10.86 & $\mathbf{2 0 1 1}$ & 2013 & \\
Interstitial pneumonia & 8.32 & $\mathbf{2 0 1 1}$ & 2013 & \\
Gene expression & 6.47 & $\mathbf{2 0 1 1}$ & 2015 & \\
Epithelial cell & 18.49 & $\mathbf{2 0 1 2}$ & 2015 & \\
Oxidative stress & 6.43 & $\mathbf{2 0 1 2}$ & 2013 & - \\
Collagen & 20.22 & $\mathbf{2 0 1 3}$ & 2015 & \\
Usual interstitial pneumonia & 7.34 & $\mathbf{2 0 1 3}$ & 2016 & \\
Emphysema & 18.54 & $\mathbf{2 0 1 4}$ & 2015 & \\
Management & 23.74 & $\mathbf{2 0 1 5}$ & 2018 & - \\
Lung cancer & 15.48 & $\mathbf{2 0 1 5}$ & 2018 & - \\
Trial & 14.24 & $\mathbf{2 0 1 5}$ & 2020 & - \\
Mice & 14.02 & $\mathbf{2 0 1 5}$ & 2017 & - \\
Growth factor & 11.40 & $\mathbf{2 0 1 6}$ & 2017 & - \\
Quality of life & 15.69 & $\mathbf{2 0 1 7}$ & 2018 & - \\
Ct & 10.13 & $\mathbf{2 0 1 7}$ & 2018 & - \\
Nintedanib & 35.48 & $\mathbf{2 0 1 8}$ & 2020 & - \\
Safety & 30.05 & $\mathbf{2 0 1 8}$ & 2020 & - \\
Efficacy & 18.20 & $\mathbf{2 0 1 8}$ & 2020 & - \\
Pathway & 11.61 & $\mathbf{2 0 1 8}$ & 2020 & - \\
\hline
\end{tabular}

Figure 8 Top 25 keywords with the strongest citation bursts. Each short line represents 1 year. The short red line represents the year in which the keywords with the citation bursts. The short blue line represents other years.

software to analyze literature on IPF published from 2011 to 2020 . The results showed the total number of annual global publications on IPF research maintained a gradual increasing trend in the past 10 years, indicating it is an increasingly important research field.

The USA had the largest number of publications and the highest centrality, and most frequently collaborated with other countries including Japan, China, England, and Germany. A strong research culture underpinned by significant public and private funding no doubt underpins this. China and South Korea also contributed many publications, but they were of low centrality and may demonstrate a lack of communication with other countries. We speculate that these countries should pay more attention to international cooperation, especially with advanced countries in this field, to promote their own progress in IPF research.

The top five institutions publishing research in the field of IPF were also from the USA, and while the University of California, San Francisco and National Jewish Health collaborated most with other institutions, which is worth learning by those seldom communicating with each other.

The American Journal of Respiratory and Critical Care Medicine was cited 4,787, making it the most cited publication. Five of the top 10 high-cited references were also published in this journal, which confirms its academic authority in the field of IPF research, reflected in its high impact factor of 17.452 .

The publication attracting the highest number of citations concerned guidelines for IPF and was authored by Raghu et al. in 2011. The paper is an official statement issued by organizations including the American Thoracic Society and European Respiratory Society, is an international evidence-based guideline on the definition, epidemiology, diagnosis, and treatment of IPF, and is generally considered as the basis of IPF research (8).

The second highest cited publication concerned a phase 3 trial investigating the efficacy and safety of nintedanib in 
IPF published in The New England Fournal of Medicine in 2014 and authored by Richeldi $e t$ al. The research proved that $150 \mathrm{mg}$ of nintedanib twice daily could significantly reduce the decline of forced vital capacity (FVC) in IPF patients $(\mathrm{P}<0.001)$ compared to placebo. The most frequent side effect of nintedanib was diarrhea, with a rate of $61.5 \%$ and $18.6 \%$ in the nintedanib and placebo group respectively, leading to less than $5 \%$ of patients discontinuing active treatment (9).

The third highest cited publication was a phase 3 trial of pirfenidone in patients with IPF also published in The New England Fournal of Medicine in 2014 and written by King et al. In that article, the authors demonstrated that oral administration of pirfenidone $2,403 \mathrm{mg} /$ day significantly delayed the decline of $\mathrm{FVC} \%$ (the percentage of the predicted FVC) $(\mathrm{P}<0.001)$, reduced the decline in the 6-minute walk distance $(\mathrm{P}=0.04)$, and improved progression-free survival $(\mathrm{P}<0.001)$ compared with placebo. Gastrointestinal and skin adverse reactions were more common in the pirfenidone group, but rarely resulted in discontinuation of treatment (10).

IPF was largely considered untreatable prior to 2014. The appearance of nintedanib and pirfenidone was a milestone in the history of treatment of the disease, and the two research articles on these drugs referred to above have been cited repeatedly as classic, highly cited, and highquality works.

According to our study, Wells, Maher, and Raghu, have contributed the most publications in the field of IPF. Wells is from the Interstitial Lung Disease Unit, Royal Brompton Hospital, and National Heart and Lung Institute, Imperial College of the UK $(11,12)$, and is the most prolific author, with 115 publications. Maher is from the Respiratory Clinical Research Facility, Royal Brompton Hospital, and National Heart and Lung Institute, Imperial College of the UK $(13,14)$, and is the second most prolific author, with 96 papers while Raghu, with 93 papers, is the third most prolific and is from the Center for Interstitial Lung Diseases, University of Washington in USA $(15,16)$. With both Wells and Maher from the Royal Brompton Hospital of UK, this institution would appear to be a major center for research in IPF. However, there was no collaboration in authorship among the top 10 authors before 2015, which shows that researchers are paying more and more attention to the importance of cooperation in the field.

The publications of Raghu were cited 2,853 times from 2011 to 2020, and that author takes the leading position in terms of both the number of articles and the degree of cooperation with other authors. As a leading authority in the field, Raghu has been the corresponding author in international IPF guidelines several times, including the Official ATS/ERS/FRS/ALAT Clinical Practice Guidelines of Idiopathic Pulmonary Fibrosis in 2011, 2015, and 2018, which were published in the American Fournal of Respiratory and Critical Care Medicine.

We identified three keyword clusters to analyze research hotspots in IPF. In cluster 1 (clinical research), in addition to "patient" and "IPF", the relative high-frequency keywords were "study", "treatment", "therapy", and "survival", suggesting the hotspots of clinical research on IPF are related to treatment and the improvement of survival rates. In clinical drug therapy, the keywords that appear most frequently were "pirfenidone" and "nintedanib". Many studies have shown that these drugs can significantly delay the decline rate of FVC, which may alleviate disease progression and reduce mortality $(9,10)$. In terms of nonpharmacological treatment, the high-frequency keyword was "lung transplantation". While lung transplantation has been shown to improve the quality of life of patients with IPF and improve the survival rate (17), with a 5-year survival rate of $53 \%$ (18), its timing and the effect of single or double lung transplantation on the prognosis of patients with IPF requires further study.

In cluster 2 (pathogenesis research), the keywords that appear more frequently were "cell", "disease", "expression", "fibrosis", "pulmonary fibrosis", "TGF beta", "pathway", and "fibroblast". At present, it is generally believed that the pathogenesis of IPF results from the interaction of multiple genetic and environmental risk factors, and among them, repeated local micro-injuries to aging alveolar epithelial cells plays a central role (19). Injured alveolar epithelial cells secrete numerous fibrogenic growth factors, including transforming growth factor- $\beta$ (TGF- $\beta$ ) and platelet-derived growth factor (PDGF) (19). TGF- $\beta$ is a strong profibrotic mediator which promotes epithelial mesenchymal transition (EMT), and activates fibroblast proliferation and transformation into myofibroblasts (20). One of the key pathways in IPF is the $\mathrm{Wnt} / \beta$-catenin pathway, which is activated by TGF- $\beta$, and is involved in EMT and fibrogenesis (21). Micro-injuries trigger abnormal epithelial-fibroblast communication, then recruit and activate myofibroblasts. Activated myofibroblasts produce matrix, resulting in a large amount of extracellular matrix accumulation and pulmonary interstitial remodeling (2).

In cluster 3 (diagnosis research), the primary keywords were "level", "control", and "biomarker". Biomarkers can 
be used for diagnosis, prognosis, and therapy response monitoring of IPF. Although many potential biomarkers have been studied, including gene expression profiles, CCL18, MMP7, and SPD, none have been verified by prospective clinical practice, and the focus of research in this domain is to verify biomarkers through multicenter collaborative prospective cohort studies (2).

Keywords burst detection was conducted to trace research hotspots and research trends (22). According to the top 25 keywords with the strongest citation bursts, early research placed emphasis on "alveolar epithelial cell", "transforming growth factor $\beta$ ", "myofibroblast", "collagen", "oxidative stress", and "endoplasmic reticulum stress", while recent research has focused on "nintedanib", "safety", "efficacy", "quality of life", and "trial". This indicates research hotspots in IPF seem to have transferred from pathogenesis to treatment, and from foundation medicine to clinical medicine.

There are many studies on the pathogenesis of IPF, and a favored pathogenesis speculates that recurrent subclinical epithelial injury on accelerated epithelial cell aging leads to the aberrant repair of injured alveoli and deposition of collagen fiber by myofibroblasts. Shortened telomeres, oxidative injury, and endoplasmic reticulum stress lead to inhibition of the proliferation of alveolar epithelial cells and stimulation of profibrotic mediator secretion (23). Nonetheless, a comprehensive understanding of IPF pathogenesis remains difficult to realize.

In recent years, the research hotspots of IPF have gradually shifted to its treatment, and significant advances have been made in pharmacotherapy. Two drugs, nintedanib and pirfenidone, are proven to be safe and effective by slowing the rate of FVC decline and potentially improving the quality of life of patients. Nintedanib is a tyrosine kinase inhibitor that targets growth factor pathways, including those downstream of fibroblast growth factor receptor, vascular endothelial growth factor receptor, and PDGF receptor (24). The most common adverse reaction of nintedanib is diarrhea. Pirfenidone has several antifibrotic effects, including the down-regulation of TGF- $\beta$, inhibition of collagen synthesis, and reduction in fibroblast proliferation (25). Common side effects of pirfenidone are anorexia, nausea, and photosensitivity. However, which of these medications provides the greatest benefit in individual patients is still unclear.

The accumulation of basic biological knowledge on fiber proliferation has promoted several new drug trials. Researchers have studied the safety, tolerability and pharmacokinetic in patients with nintedanib and pirfenidone combination therapy versus those treated with nintedanib alone, which suggested that nintedanib and pirfenidone combination therapy had manageable levels of safety and tolerability (ClinicalTrials.gov Identifier: NCT02579603). Pamrevlumab is an antibody targeting connective- tissue growth factor, which has shown promise in delaying the decline of FVC compared with placebo. At present, a phase 3 , randomized, placebo-controlled, multi-center trial to evaluate its efficacy and safety is ongoing (ClinicalTrials.gov Identifier: NCT03955146).

Previously, IPF was considered in a completely untreatable. Progress has been made in the research and development of pulmonary anti-fibrosis drugs, and the emergence of nintedanib and pirfenidone has provided a milestone breakthrough in the history of IPF treatment. Whereas pirfenidone and nintedanib are currently recommended for patients with mild to moderate pulmonary dysfunction with IPF, their benefit in severe disease is unclear, and adverse reactions may limit their use in some patients. Moreover, the cost of each drug is estimated to more than $\$ 100,000$ annually (24), which may further limit their application for many patients. Above all, these drugs can only delay the decline rate of FVC, and are unable to reverse its progression, and the survival rate of patients remains very low. Lung transplantation can prolong survival, but the high technical requirements, uncertain survival rate after transplantation, donor lung scarcity, high cost, and other factors objectively limit its implementation in clinical practice.

According to the results of literature analysis, we could conclude that the future research direction will focus on conducting multi-center randomized controlled trials to explore and evaluate the new therapeutic drugs for IPF. Many trials are needed to screen drugs that may halt collagen deposition, have less adverse reactions, and are affordable. A future treatment strategy for IPF may be a combination of multiple drugs targeting different aspects of pulmonary fibrosis, perhaps reducing the need for lung transplantation.

\section{Conclusions}

This study utilized information visualization software to evaluate the contributions of countries, institutions, journals and authors, to analyze research hotspots on IPF research over the past decade. The USA took the leading position in this field by contributing the most publications, and the 
top five research institutions with the largest number of publications were also from that country. The American Fournal of Respiratory and Critical Care Medicine attracted the most citations, and had an incontrovertible academic impact, with five of the top 10 high-cited references published in this journal. Raghu was the academic authority in this domain in terms of both the number of publications and the most cited publications. The results also showed that the current research hotspot in the field of IPF is drug therapy largely involving the medications nintedanib and pirfenidone. Future research directions focusing on multi-center randomized controlled trials to explore and evaluate new therapeutic drugs for IPF will rely on the close cooperation of multiple institutions and scholars. Our research identified the authoritative institutions and scholars, indicated the high-cited literatures, and analyzed hotspots and future directions in IPF research. These findings will enable researchers to identify leading institutions and scholars, to seek academic collaboration with leaders in the field, and to keep abreast of current and future directions in IPF research.

\section{Acknowledgments}

Funding: This work was supported by grants from the China Postdoctoral Science Foundation (grant No. 2019M661163) and the Department of Science and Technology of Liaoning Province, China (grant No. 2019-BS-280).

\section{Footnote}

Conflicts of Interest: All authors have completed the ICMJE uniform disclosure form (available at http://dx.doi. org/10.21037/apm-21-1836). All authors report funding from the China Postdoctoral Science Foundation (grant No. 2019M661163) and the Department of Science and Technology of Liaoning Province, China (grant No. 2019BS-280). The authors have no other conflicts of interest to declare.

Ethical Statement: The authors are accountable for all aspects of the work in ensuring that questions related to the accuracy or integrity of any part of the work are appropriately investigated and resolved.

Open Access Statement: This is an Open Access article distributed in accordance with the Creative Commons Attribution-NonCommercial-NoDerivs 4.0 International
License (CC BY-NC-ND 4.0), which permits the noncommercial replication and distribution of the article with the strict proviso that no changes or edits are made and the original work is properly cited (including links to both the formal publication through the relevant DOI and the license). See: https://creativecommons.org/licenses/by-nc-nd/4.0/.

\section{References}

1. Raghu G, Remy-Jardin M, Myers JL, et al. Diagnosis of idiopathic pulmonary fibrosis. An official ATS/ERS/JRS/ ALAT clinical practice guideline. Am J Respir Crit Care Med 2018;198:e44-68.

2. Richeldi L, Collard HR, Jones MG. Idiopathic pulmonary fibrosis. Lancet 2017;389:1941-52.

3. Wakwaya Y, Brown KK. Idiopathic pulmonary fibrosis: epidemiology, diagnosis and outcomes. Am J Med Sci 2019;357:359-69.

4. Hewlett JC, Kropski JA, Blackwell TS. Idiopathic pulmonary fibrosis: Epithelial-mesenchymal interactions and emerging therapeutic targets. Matrix Biol 2018;7172:112-27.

5. Zhang S, Mao G, Crittenden J, et al. Groundwater remediation from the past to the future: A bibliometric analysis. Water Res 2017;119:114-25.

6. Xing D, Zhao Y, Dong S, et al. Global research trends in stem cells for osteoarthritis: a bibliometric and visualized study. Int J Rheum Dis 2018;21:1372-84.

7. Pu QH, Lyu QJ, Su HY. Bibliometric analysis of scientific publications in transplantation journals from Mainland China, Japan, South Korea and Taiwan between 2006 and 2015. BMJ Open 2016;6:e011623.

8. Raghu G, Collard HR, Egan JJ, et al. An official ATS/ ERS/JRS/ALAT statement: idiopathic pulmonary fibrosis: evidence-based guidelines for diagnosis and management. Am J Respir Crit Care Med 2011;183:788-824.

9. Richeldi L, du Bois RM, Raghu G, et al. Efficacy and safety of nintedanib in idiopathic pulmonary fibrosis. $\mathrm{N}$ Engl J Med 2014;370:2071-82.

10. King TE Jr, Bradford WZ, Castro-Bernardini S, et al. A phase 3 trial of pirfenidone in patients with idiopathic pulmonary fibrosis. N Engl J Med 2014;370:2083-92.

11. Lynch DA, Sverzellati N, Travis WD, et al. Diagnostic criteria for idiopathic pulmonary fibrosis: a Fleischner Society White Paper. Lancet Respir Med 2018;6:138-53.

12. Cottin V, Hirani NA, Hotchkin DL, et al. Presentation, diagnosis and clinical course of the spectrum of progressive-fibrosing interstitial lung diseases. Eur Respir 
Rev 2018;27:180076.

13. Spagnolo P, Balestro E, Aliberti S, et al. Pulmonary fibrosis secondary to COVID-19: a call to arms? Lancet Respir Med 2020;8:750-2.

14. Distler O, Assassi S, Cottin V, et al. Predictors of progression in systemic sclerosis patients with interstitial lung disease. Eur Respir J 2020;5 5:1902026.

15. Podolanczuk AJ, Noth I, Raghu G. Idiopathic pulmonary fibrosis: prime time for a precision-based approach to treatment with $\mathrm{N}$-acetylcysteine. Eur Respir J 2021;57:2003551.

16. Raghu G, Remy-Jardin M, Ryerson CJ, et al. Diagnosis of hypersensitivity pneumonitis in adults. An official ATS/ JRS/ALAT clinical practice guideline. Am J Respir Crit Care Med 2020;202:e36-69.

17. Singer JP, Katz PP, Soong A, et al. Effect of lung transplantation on health-related quality of life in the era of the lung allocation score: a U.S. prospective cohort study. Am J Transplant 2017;17:1334-45.

18. Valapour M, Lehr CJ, Skeans MA, et al. OPTN/SRTR 2016 annual data report: lung. Am J Transplant 2018;18 Suppl 1:363-433.

Cite this article as: Ma Y, Zhou R, Wu Q. Global research hotspots and research trends on idiopathic pulmonary fibrosis: a bibliometric and visualization analysis. Ann Palliat Med 2021;10(8):9057-9068. doi: 10.21037/apm-21-1836
19. Sgalla G, Iovene B, Calvello M, et al. Idiopathic pulmonary fibrosis: pathogenesis and management. Respir Res 2018;19:32.

20. Grimminger F, Günther A, Vancheri C. The role of tyrosine kinases in the pathogenesis of idiopathic pulmonary fibrosis. Eur Respir J 2015;45:1426-33.

21. King TE Jr, Pardo A, Selman M. Idiopathic pulmonary fibrosis. Lancet 2011;378:1949-61.

22. Dong G, Li R, Yang W, et al. Microblog burst keywords detection based on social trust and dynamics model. Chinese Journal of Electronics 2014;23:695-700.

23. Selman M, Pardo A. Revealing the pathogenic and agingrelated mechanisms of the enigmatic idiopathic pulmonary fibrosis. an integral model. Am J Respir Crit Care Med 2014;189:1161-72.

24. Lederer DJ, Martinez FJ. Idiopathic pulmonary fibrosis. N Engl J Med 2018;378:1811-23.

25. Kolb M, Bonella F, Wollin L. Therapeutic targets in idiopathic pulmonary fibrosis. Respir Med 2017;131:49-57.

(English Language Editor: B. Draper) 


\section{Supplementary}

Table S1 The 217 keywords with at least 218 occurrences

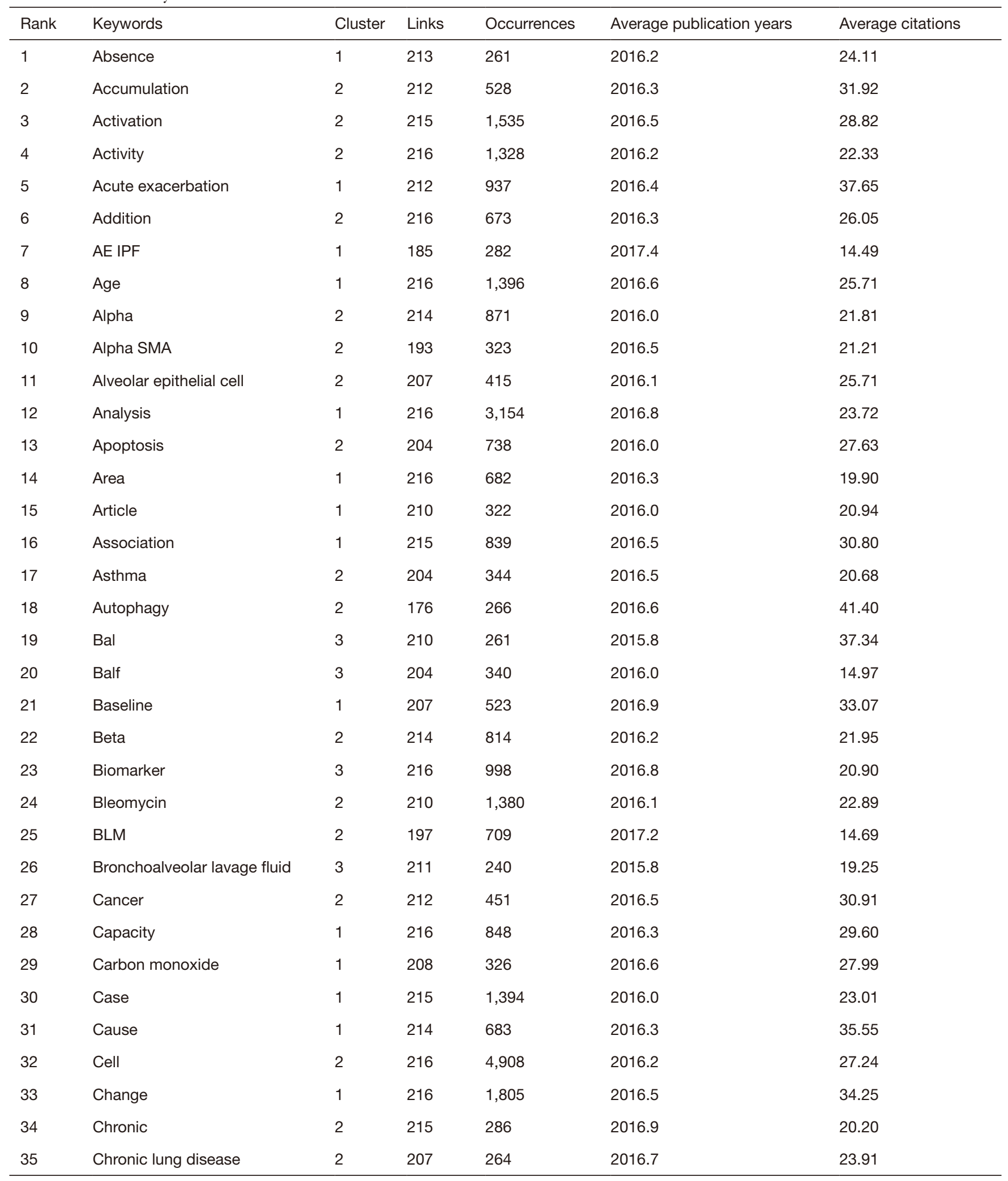

Table S1 (continued) 
Table S1 (continued)

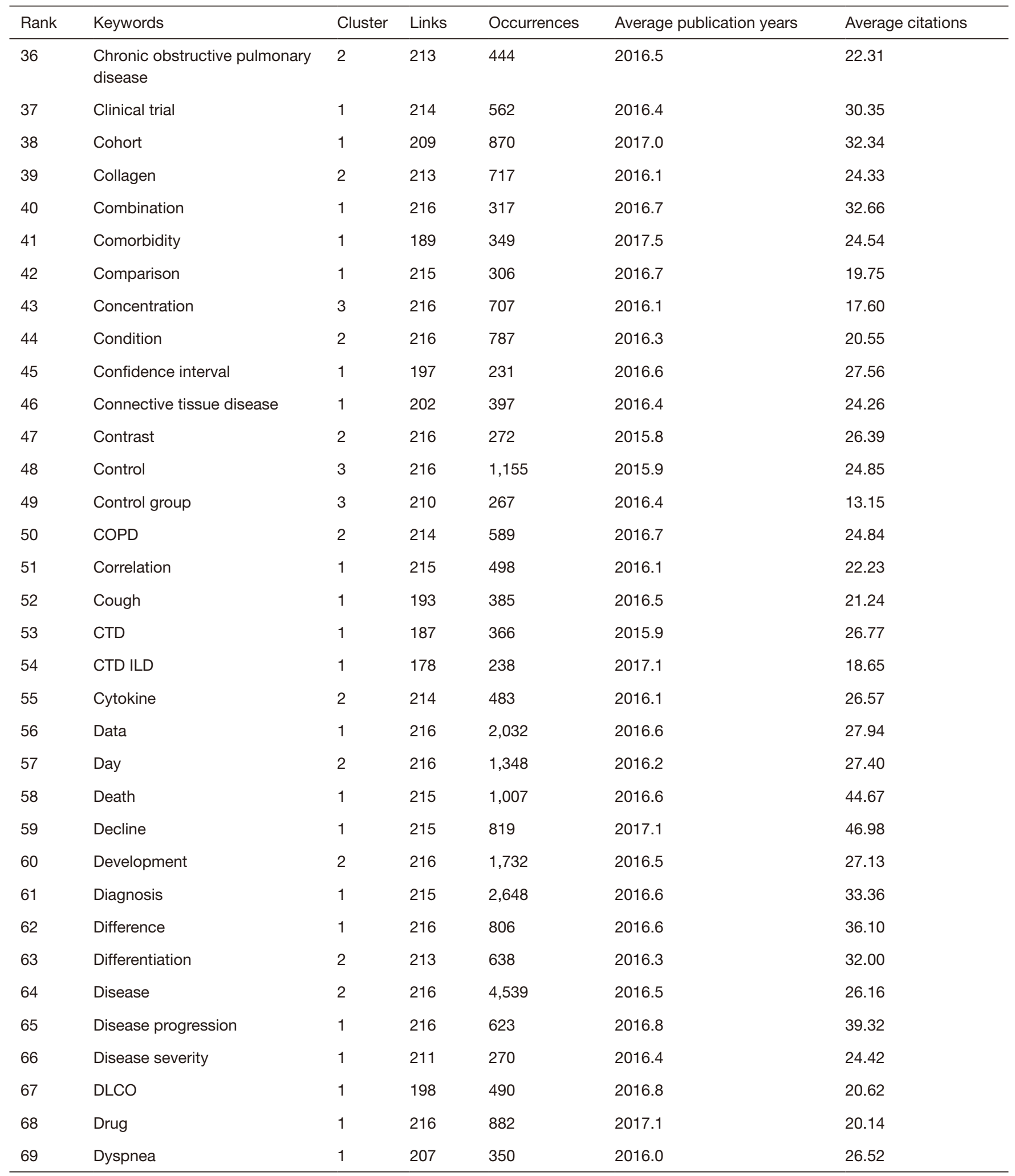

Table S1 (continued) 
Table S1 (continued)

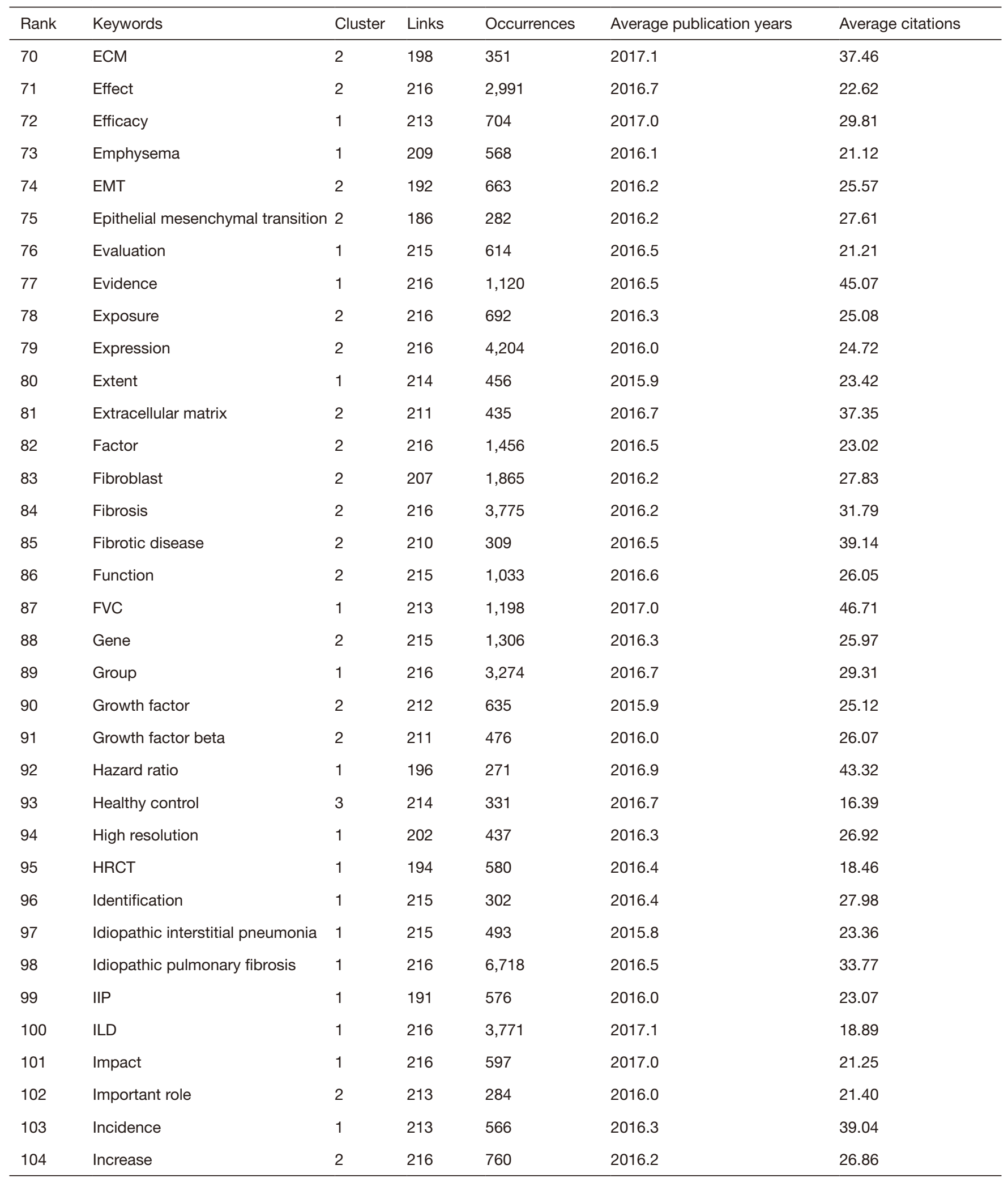

Table S1 (continued) 
Table S1 (continued)

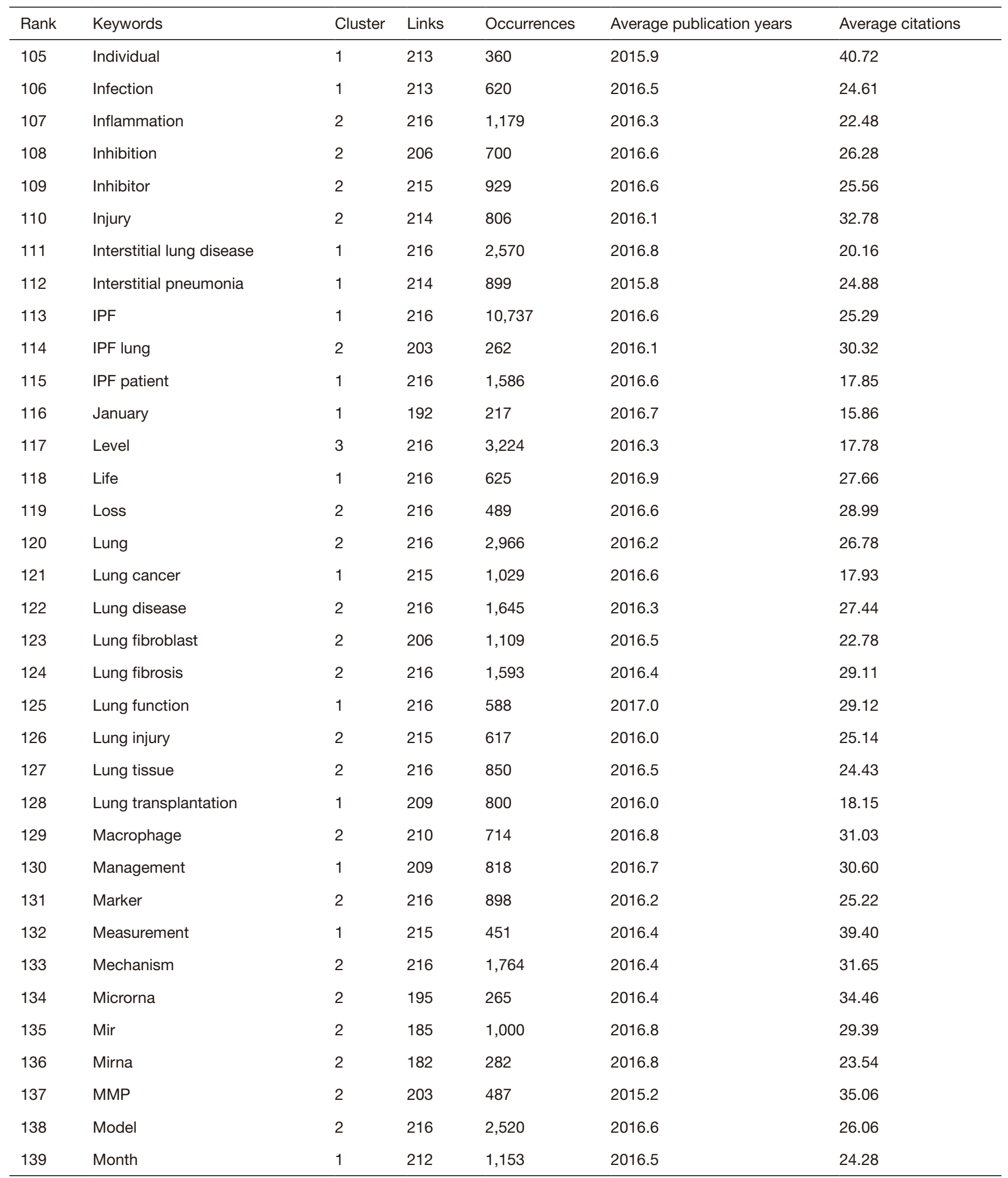

Table S1 (continued) 
Table S1 (continued)

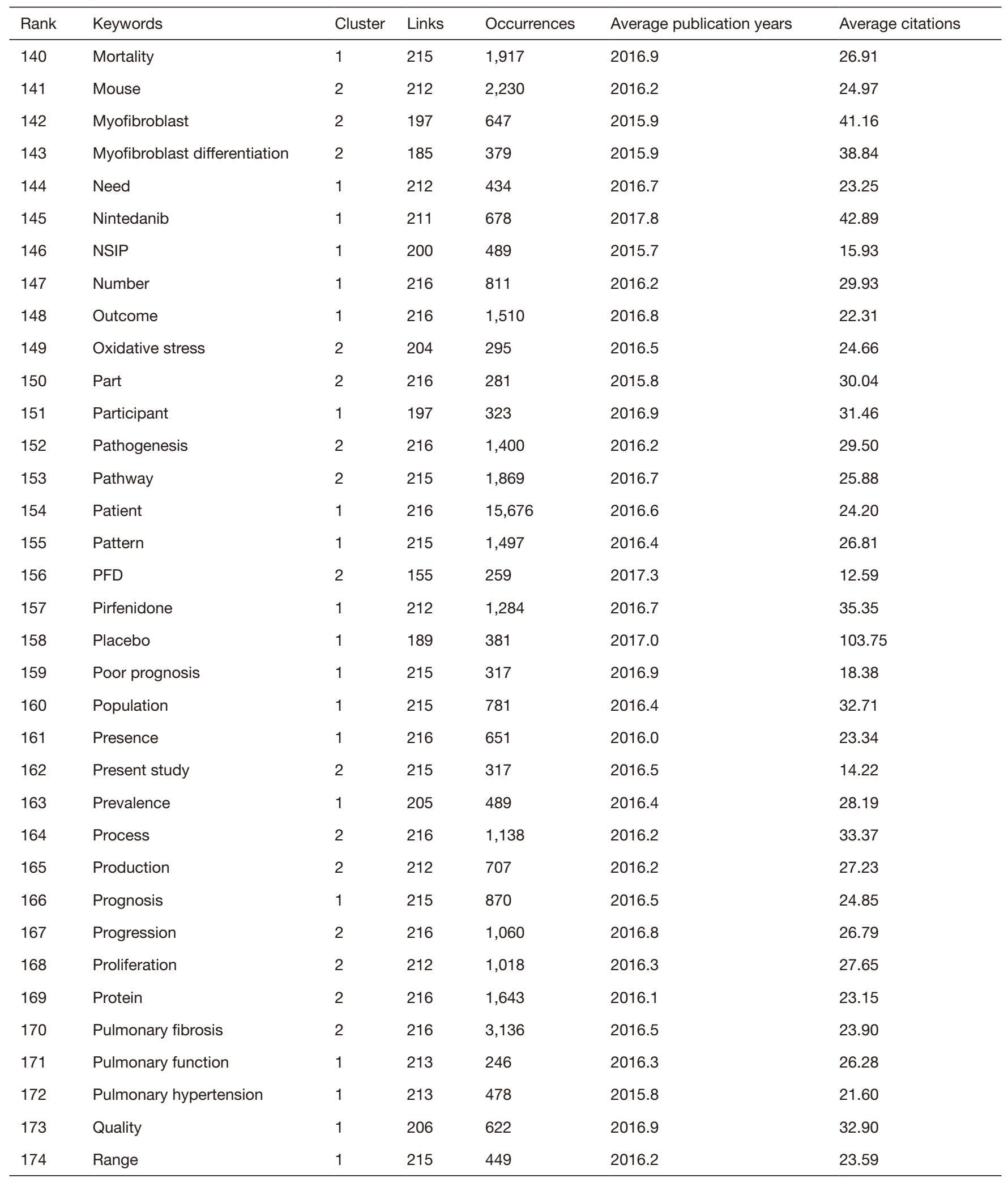

Table S1 (continued) 
Table S1 (continued)

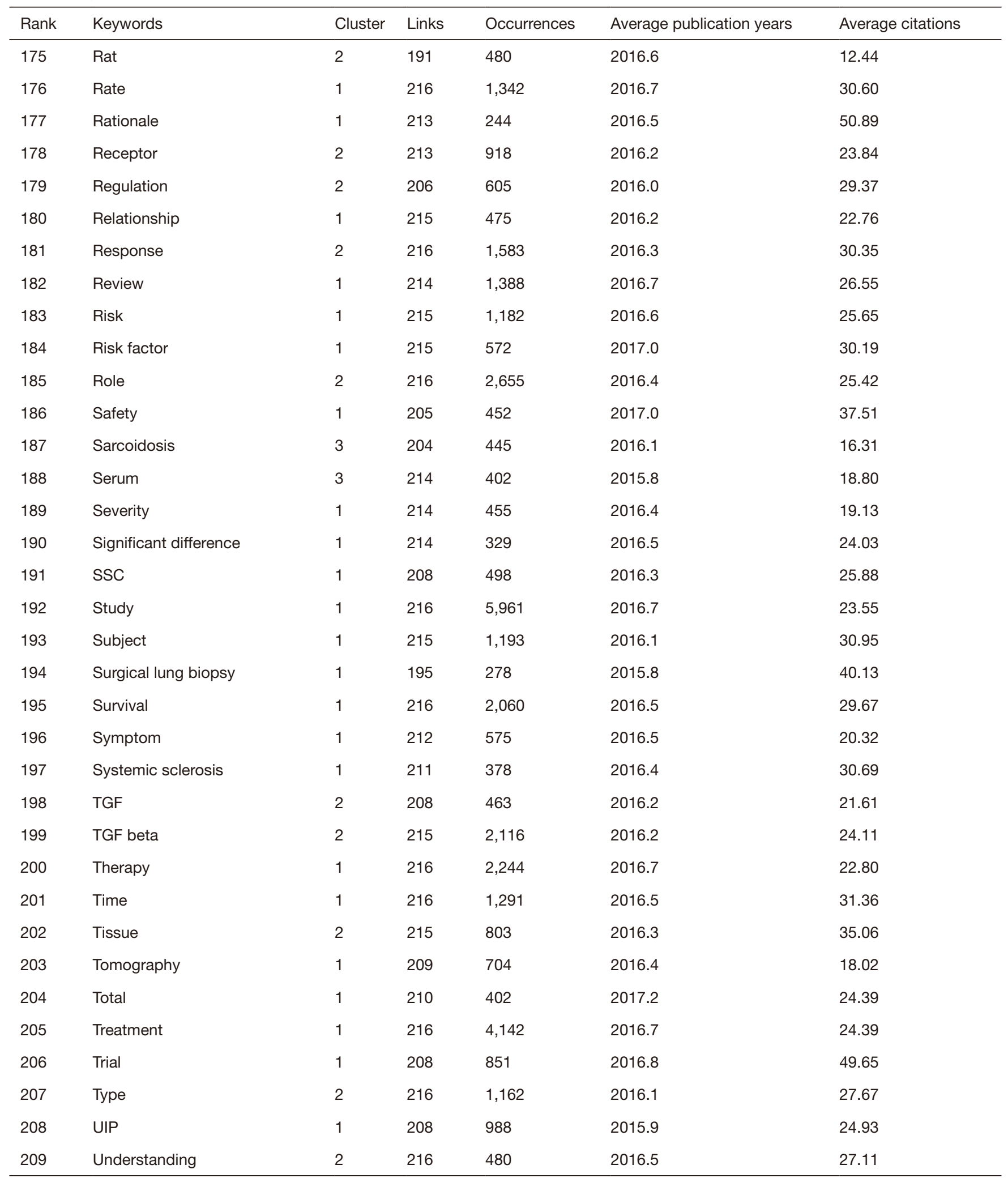

Table S1 (continued) 
Table S1 (continued)

\begin{tabular}{lllllll}
\hline Rank & Keywords & Cluster & Links & Occurrences & Average publication years & Average citations \\
\hline 210 & Use & 1 & 216 & 913 & 2016.9 & 21.54 \\
211 & Usual interstitial pneumonia & 1 & 212 & 479 & 2015.9 & 26.39 \\
212 & Value & 1 & 216 & 806 & 2016.7 & 22.09 \\
213 & Vital capacity & 1 & 213 & 606 & 2016.7 & 37.86 \\
214 & Vitro & 2 & 208 & 539 & 2016.4 & 26.26 \\
215 & Vivo & 2 & 189 & 272 & 2016.6 & 24.90 \\
216 & Week & 1 & 215 & 649 & 2016.9 & 51.69 \\
217 & Year & 1 & 216 & 2,077 & 2016.6 & 26.34 \\
\hline
\end{tabular}

